(ZK370 and C30A5) compiled by the Nematode Genome Sequencing Project ${ }^{2}$. We have also published an alignment of this transposase with those of the insect elements ${ }^{3}$. In addition, M. Sedensky and P. Morgan (manuscript in preparation) have found a quite distinct mariner in C. elegans. These two mariners do not cluster with insect mariners and represent additional subfamilies. Finally, T. Langin, P. Capy and M. J. Daboussi (manuscript in preparation) have found mariner elements in a fungus. These parasitic genes are therefore of great antiquity, and have infiltrated the genomes of many organisms.

Hugh M. Robertson

Department of Entomology,

University of Illinois,

Urbana-Champaign,

Illinois 61801, USA

1. Robertson, H. M. \& MacLeod, E. G. Insect molec. Biol. (in the press).

. Sulston, J. et al. Nature 356, 37-41 (1992)

3. Robertson, H. M., Lampe, D. J. \& MacLeod, E. G. Nucleic Acids Res. 20, 6409 (1992)

\section{HIV quiet but not silent}

SIR - Much attention has been focused on the site and quantification of HIV infection in the early stages, before significant immunosuppression. It has been implied that asymptomatic HIV infection is

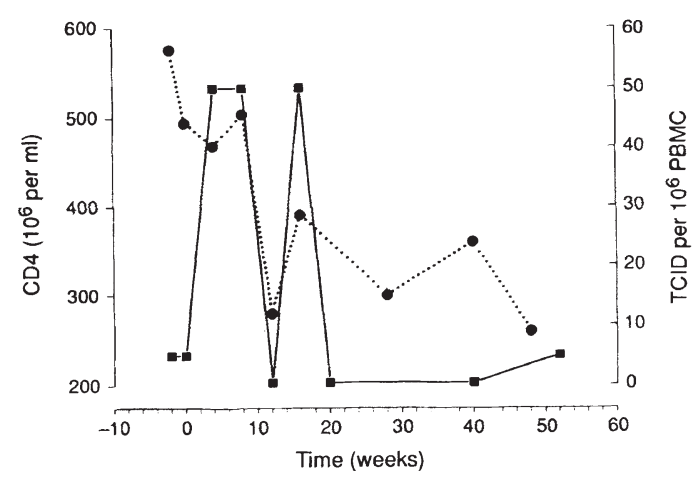

Fluctuations in infectious viral load in PBMC during asymptomatic HIV infection. Circles, CD4 cells, squares, tissue-culture infectious dose (TCID).

clinically silent, and that viral replication may be restricted largely to the lymph nodes $^{1,2}$.

Although early HIV infection is often described as asymptomatic, it is far from silent clinically or virologically. In practice, all HIV-positive people have some degree of fluctuating lymphadenopathy throughout the asymptomatic stage, which may or may not be sufficiently pronounced to be termed persistent generalized lymphadenopathy ${ }^{3}$. Hypergammaglobulinaemia is also detectable early in the course of HIV infection, and thrombocytopaenia may occur throughout this period. Even when the CD4 count exceeds $500 \times 10^{6} \mathrm{l}^{-1}$, HIV-positive people may experience recurrent folliculitis, especially in the beard area, seborrhoeic dermatitis on the face and chest and generalized xeroderma; pityriasis versicolor infection is common.

1. Pantaleo, G. etal. Nature 362, 355-358 (1993)

2. Embretson, etal Nature 362, 359-362 (1993).

3. Centers for Disease Control Morbid. Mortal. Wkly Rep. 31 248-251(1982)

4. Weber, J. \& Pinching, A. J. in The Management of AIDS Patients (eds. Miller, D., Weber, J. \& Green, J.) 1-35 (Macmillan, London, 1986).

5. Bieniasz, P. et al. AIDS 7, 803-807 (1993).
Although these phenomena can be thought of as minor opportunist infections, "asymptomatic" subjects also report bouts of self-limiting night sweats, generally without objective fever, but often associated with profound lethargy and fatigue ${ }^{4}$.

These episodes may be temporally related to the peaks of infectious virus load seen in the peripheral blood lymphocytes. Although viral burden may be greatest in lymph nodes early in the course of HIV infection, infectious virus can be recovered from peripheral blood mononuclear cells (PBMC) in most HIVpositive patients throughout the course of infection, from primary infection onwards ${ }^{5}$. The level of infectious virus load in PBMCs fluctuates considerably over time in asymptomatic subjects (see figure), although the full significance of these fluctuations is still unclear.

HIV leads to systemic manifestations throughout the course of infection, and although these conditions may not be life-threatening, they are far from silent. Jonathan Weber

Myra McClure

Department of Communicable Diseases,

St Mary's Hospital Medical School,

London W2 1NY, UK

\section{Mouse coat colour reconsidered}

SIR - Jackson's interesting discussion ${ }^{1}$ in News and Views of coat-colour genes proposes an unnecessarily narrow hypothesis and ignores a more likely one. Jackson depicts the agouti protein as a competitive antagonist that binds to the melanocyte-stimulating hormone receptor (MSH-R), but fails to consider an alternative possibility, that the agouti protein acts instead as an agonist on a separate receptor, triggering a pathway that opposes downstream effects of the MSH-R. Ignoring the alternative hy- pothesis runs the risk of overlooking a potentially important determinant of coat colour and inadequately explains the pleiotropic effects of a dominant mutation at the agouti locus.

The MSH-R and the agouti locus control coat colour via opposing effects on a bifurcated biochemical pathway that converts tyrosine to either phaeomelanin (yellow coat) or eumelanin (black coat). Activation of the MSH-R promotes synthesis of eumelanin ${ }^{2}$, while the agouti protein enhances production of phaeomelanin $^{3}$ (the default pathway). The agouti protein could act by binding directly to the MSH-R, as Jackson suggests. Alternatively, a distinct receptor for the agouti protein could activate a signalling pathway that independently regulates melanin production in the target cell. Push-pull regulation by opposing hormones is common in nature (for example heart rate, glycogenolysis). The notion of a naturally occurring, physiologically relevant competitive antagonist acting on a cell surface receptor is intriguing, but to our knowledge, without precedent.

Ubiquitous overproduction of the agouti protein in mice with a dominant yellow mutation at the agouti locus causes obesity, diabetes and tumours in addition to a yellow coat colour ${ }^{3}$. Injection of MSH can override the effect of this mutation on coat colour ${ }^{4}$ but has not been shown to counteract the other effects. To explain this Jackson broadens the hypothetical antagonist activity of the agouti protein to block other (non-MSH) receptors ${ }^{1}$. A simpler model would allow the agouti protein to induce obesity, diabetes and tumours by activating its own receptor.

If the agouti protein has a distinct receptor, why has its gene eluded coatcolour geneticists? It is well known that genes that are necessary for viability, or that encode proteins with redundant functions, can be refractory to traditional linkage analysis. This is probably why the genes for adenylylcyclase and $\mathrm{G}_{\alpha \mathrm{s}}$ are not defined by coat-colour loci although the protein products of both genes are undoubtedly necessary for MSH-driven coat darkening.

Studies with purified agouti protein will eventually show whether it is a competitive antagonist or acts on its own receptor. Until then it seems prudent to consider all the options.

\section{Bruce R. Conklin}

Henry R. Boume

Departments of Pharmacology and

Medicine and Programs in Biomedical

Sciences and Cell Biology,

University of California, San Francisco,

California 94143-0450, USA

1. Jackson, I. J. Nature $362,587-588$ (1993).

2. Robbins, L. S. etal. Cell 72, 827-834 (1993)

3. Bultman, S. J., Michaud, E. J. \& Woychik, R. P. Cell 71, 1195-1204 (1992).

4. Jackson, I. J. BioEssays 13, 439-446 (1991) 